\title{
Review of: "Rationale and evidence on the use of tocilizumab in COVID-19: a systematic review"
}

Xinyi Xia

Potential competing interests: The author(s) declared that no potential competing interests exist.

\section{Can Tocilizumab be trusted to treat COVID-19 patients?}

Weijun Jiang ${ }^{1}$, Xinyi Xia ${ }^{1,} 2,3$,

1, COVID-19 Research Center, Institute of Laboratory Medicine, Jinling Hospital, Nanjing University School of Medicine, The First School of Clinical Medicine, Southern Medical University, Nanjing 210002, Jiangsu, China

2, Joint Expert Group for COVID-19, Department of Laboratory Medicine and Blood Transfusion, Wuhan Huoshenshan Hospital, Wuhan 430100, Hubei, China

3, Department of Laboratory Medicine and Blood Transfusion, Wuhan Huoshenshan Hospital, Wuhan 430100, Hubei, China

Corresponding author: Xinyi Xia e-mail: xinyixia@nju.edu.cn

Dear editor $\square$

Cortegiani et al. ${ }^{1}$ recently conducted a systematic review, "Rationale and evidence on the use of tocilizumab in COVID-19: a systematic review," published in this article of Pulmonology. They identified three indirect pre-clinical and 28 clinical studies, including 5776 patients with coronavirus disease 2019 (COVID-19, 13 with a comparison group, 15 single-arm). They reported the evidence and evaluated the quality of evidence concerning the use of Tocilizumab in patients with COVID-19. However, due to the early publication of the article, new high-quality articles are constantly published. We want to complement the discussion on the evidence of tocilizumab use in patients with COVID-19.

\section{When should the patient use Tocilizumab?}

In the randomized controlled RECOVERY trial, Peter W Horby et al. ${ }^{2}$ reported hospitalized COVID-19 patients with hypoxia and systemic inflammation, and Tocilizumab improved survival and other clinical outcomes. Olivier Hermine et al. $^{3}$ said Tocilizumab might have reduced the risk of noninvasive ventilation, mechanical ventilation, or death by day 14 . Anthony C. Gordon et al. ${ }^{4}$ also reported that treatment with Tocilizumab improved outcomes, including survival in critically ill patients with Covid-19 receiving organ support in ICUs. Jingwen Peng et al. ${ }^{5}$ also found that Tocilizumab significantly decreased mortality in COVID-19 patients in the meta-analysis. Weijun Jiang et al. ${ }^{6}$ also found Tocilizumab significantly reduced mortality in COVID-19 patients with no increased discharge, secondary infection risk, adverse events, and mechanical ventilation in a meta-analysis. Our data suggest that clinicians should pay attention to tocilizumab therapy as an effective and safe treatment for COVID-19 patients. 
However, some randomized clinical trials reported Tocilizumab did not result in significantly better clinical status or lower mortality than placebo at 28 days. Viviane $C$ Veiga et al. ${ }^{7}$ found that Tocilizumab plus standard care was not superior to usual care alone in improving clinical outcomes at 15 days. It might increase mortality in patients with severe or critical COVID-19.

\section{How much is Tocilizumab used does best?}

There is also concern about the dosage of Tocilizumab. We found the tocilizumab dose (i.e., <400mg, $400 \mathrm{mg}, 400 \mathrm{mg}-800 \mathrm{mg}$, and $8 \mathrm{mg} / \mathrm{kg}$ ) in randomized controlled trials, retrospective observational studies, case-control studies, and cohort studies. All participants received placebo plus standard treatment or only standard treatments including hydroxychloroquine and antiviral therapy, including lopinavir/ritonavir, antimicrobial agents, and corticosteroids 8,9. These all affect the results of the experiment in all studies.

\section{What are the adverse events associated with Tocilizumab?}

The use of Tocilizumab is not harmless since it may predispose patients to the development of secondary infections. Jingwen Peng et al. ${ }^{5}$ found that tocilizumab therapy significantly increased the risk of fungal coinfections in COVID-19 patients, eight observational studies (601 cases and 783 controls), in the overall analysis $(\mathrm{OR}=2.02,95 \% \mathrm{Cl}=1.05-3.90, P=0.036)$. We suggest that clinicians pay attention to tocilizumab therapy as an effective and safe treatment for COVID-19 patients.

\section{Conflicts of interest}

All authors declare that they have no conflicts of interest in the work.

\section{References}

1. Cortegiani A, Ippolito M, Greco M, Granone V, Protti A, Gregoretti C, et al. Rationale and evidence on the use of tocilizumab in COVID-19: a systematic review. Pulmonology. 2021;27:52-66.

2. Group RC, Horby PW, Pessoa-Amorim G, Peto L, Brightling CE, Sarkar R, et al. Tocilizumab in patients admitted to hospital with COVID-19 (RECOVERY): preliminary results of a randomised, controlled, open-label, platform trial. medRxiv. 2021:2021.02.11.21249258.

3. Hermine O, Mariette X, Tharaux PL, Resche-Rigon M, Porcher R, Ravaud P. Effect of Tocilizumab vs Usual Care in Adults Hospitalized With COVID-19 and Moderate or Severe Pneumonia: A Randomized Clinical Trial. JAMA internal medicine. 2021;181:32-40.

4. Gordon AC, Mouncey PR. Interleukin-6 Receptor Antagonists in Critically III Patients with Covid19. $2021 ; 384: 1491-502$.

5. Peng J, Fu M, Mei H, Zheng H, Liang G, She X, et al. Efficacy and secondary infection risk of tocilizumab, sarilumab and anakinra in COVID-19 patients: A systematic review and meta-analysis. 2021:e2295.

6. Jiang W, Li W, Wu Q, Han Y, Zhang J, Luo T, et al. Efficacy and Safety of Tocilizumab Treatment COVID-19 Patients: A Case-Control Study and Meta-Analysis. 2021;10:1677-98.

7. Veiga VC, Prats J, Farias DLC, Rosa RG, Dourado LK, Zampieri FG, et al. Effect of tocilizumab on clinical outcomes at 15 days in patients with severe or critical coronavirus disease 2019: randomised controlled trial. BMJ (Clinical research ed.). 2021;372:n84. 
8. Salvarani C, Dolci G, Massari M, Merlo DF, Cavuto S, Savoldi L, et al. Effect of Tocilizumab vs Standard Care on Clinical Worsening in Patients Hospitalized With COVID-19 Pneumonia: A Randomized Clinical Trial. JAMA internal medicine. 2021;181:24-31.

9. Rosas IO, Bräu N, Waters M, Go RC, Hunter BD, Bhagani S, et al. Tocilizumab in Hospitalized Patients with Severe Covid-19 Pneumonia. 2021;384:1503-16. 